\title{
Social recognition provision patterns in professional Q\&A forums in Healthcare and Construction
}

\author{
Corresponding Author: Dr. Kai Pata, Ph.D.
}

\section{Corresponding Author's Institution: Tallinn University}

First Author: Kai Pata, Ph.D.

Order of Authors: Kai Pata ${ }^{a}$, Ph.D.; Patricia Santos ${ }^{b}$, Ph.D.; Joanna Burchert ${ }^{c}$, Ph.D.

${ }^{a}$ Center for Educational Technology, Institute of Informatics, Tallinn University, Tallinn, Estonia

${ }^{\mathbf{b}}$ Arts and Cultural Industries Department, University of the West of England, Bristol

c Institut Technik und Bildung, University of Bremen

E-mail: kpata@tlu.ee, Patricia.Santosrodriguez@uwe.ac.uk, burchert@uni-bremen.de

\begin{abstract}
For some decades, professional Q\&A forums have been used as a mainstream way of sharing practices between novices and experts. Several forums have had time to develop their own communities and habits, which made them a suitable place to explore patterned epistemic practices. In this paper we look at the social recognition, help seeking and and informal learning patterns in communities of practice; our aim is to use the corresponding outputs to scaffold technology supported informal learning. We analysed professional discussion forums in two countries (UK and Germany) in two different sectors (healthcare and construction). We identified a set of interrelated patterns that are used for socially verifying and maturing rules and guidelines, solving problems, introducing new practices and triggering learning. Some particular social recognition and learning trends common in Healthcare and Construction sector Q\&A forums are highlighted. We discuss epistemic practice pattern networks for developing scaffolds to enhance the quality of informal learning in workplace environments in an integrated way. We suggest and validate empirically a model of social recognition provision in Q\&A forums.
\end{abstract}

\section{Keywords}

workplace learning, epistemic practice patterns, social recognition, Q\&A forum, social networking, community of practice

\section{Introduction}

One of the goals in lifelong learning policies and initiatives is scaling up the incidental and informal learning (Tynjala, 2008; Ley et al., 2014) that can be done by noticing, recording, sharing, and formally and socially recognizing those practices acquired at on the job training (Werquin, 2010), and uniting individual informal learning and organizational learning practices into an evolving knowledge creation and conversing systems (e.g. Nonaka \& Takeuchi, 1995; Tammets et al., 2014). Recognition practices - 
both social and formal - play a key role in this knowledge conversion that makes organizations and practice communities responsive to internal and external changes. Responsiveness as system's adaptation to environmental change is influenced by the distributed intelligence and dispersed learning processes carried out within organization or community to mitigate negative threats or capitalize on positive opportunities: knowledge exchanges among individuals, the aggregation of individual actions to increase the visibility of joint practices and issues, recognition of common responses to environmental demands and interpretation of external and internal changes generated by shared environment (Daft and Weick, 1984, Goodstein, 1995, Brusoni et al., 2001, Jacobs, 2003, Bray et al., 2007).

The informal learning practices evolved in professional communities have remained largely an unexplored territory. Lately, in EU 7th Framework project Learning Layers (http://learning-layers.eu) several empirical interview and design-based research studies have revealed some common practices among Healthcare and Construction stakeholders (Ley et al., 2014). In this paper we explore what role the recognition practices have in informal practice communities in the Q\&A forums in Healthcare and Construction sectors. For some decades, professional Q\&A forums have been used as a mainstream way of sharing informally practices between novices and experts. Several forums have had time to develop their own communities and habits, which makes them a suitable place to explore patterned epistemic practices (Roepstorff, et al., 2010) induced by technological environments. The technology development for scaling up informal learning at work has to make use of the synergy from different interrelated practices to support informal learning in an integrated way. Particularly our approach is investigating patterns as associated networks of practices and detecting patterns that are common across different informal learning communities. Knowing such patterned epistemic practices would help developing scaffolding elements that can advance communities in using useful patterns of epistemic practices and thus aid the informal learning and shared knowledge-building in practice communities.

The main goals of the study presented in this paper are: identifying patterned epistemic practices as networks of practices in informal help-seeking communities in Construction and Healthcare Q\&A forums; developing and validating a model of social recognition provision in professional Q\&A forums; and suggesting the further application possibilities of using social recognition provision in professional Q\&A forums.

\section{Related work}

\subsection{Patterned epistemic practices}

Roepstorff et al. (2010) argue that human practices are characterized by particular patterns, and that participating in these patterns orders how people perceive and act in particular group and context specific ways. By definition, a pattern is any regularity that organises what we see in a consistent, regular manner. Alexander and associates (1977) define design patterns as easily recognisable visible/explicit part of a solution to a problem in a field of interest: patterns are generalisations of solutions for a problem. Patterns can appear like visible macro-structures of processes or structures: i) event sequences in distributed systems (e.g. learning paths, discourse act sequences), ii) semantic knowledge structures (e.g. associated tags), as iii) functional (e.g. design solutions) or iv) behavioural (e.g. social network visualisations of interaction) compositions (Pata \& Bardone, 2014).

Roepstorff, et al. (2010) point to the necessity of abandoning 'culture' as an analytically meaningful concept and moving to 'patterns of practice' instead because this shift from culture to patterns of practice mirrors in important ways the shift from individual to distributed or embodied cognition (Hutchins, 
2010). Culture propagates itself with patterns and pattern systems (Alexander et al., 1977). Patterns tend to focus on the interactions between the physical form of the built environment, and the way in which that inhibits or facilitates various sorts of personal and social behaviour within it (Alexander et al., 1977). The patterned epistemic practices concept assumes that people interact and thereby actualize concrete, shared material-discursive environments. For example, we can assume that Q\&A forum communities as sociotechnical systems may facilitate or constrain some patterns to emerge. The patterned epistemic practices concept takes up the basic insight that any action is embedded in three heuristically different "environments" - normative orders, social dynamics and material conditions (Parsons, 1937) where actors are confronted with the challenge to to solve practical problems (Alexander, 1988), and must interactively with others interpret, understand, and strategize in concrete situations particular ways of doing things as joint activities (Roepstorff, et al., 2010). Patterns of practice are shaped by neural networks as well as belief systems and normative orders (Roepstorff, et al., 2010).

Patterns emerge as the contingent result of all the occasions there have been to renew or enrich, or to maintain the stock of this problem's solutions, using the remains of previous constructions or destructions from individuals (Pata \& Bardone, 2014). Culturally, each pattern exists as an emergent niche in the ecosystem in which the environment becomes anticipated and ecologically encultured due to many persons' activities (Pata \& Bardone, 2014). Being the member of a certain practice culture in a community, workplace learners are tuned to noticing and enacting some and ignoring the other affordances of the environment detecting them as as patterns to be followed. The trivial understanding of the usage of patterned epistemic practices in the practice communities is, that copying a patterned practice as a defined sequence of practices it can be used as a template to guarantee an expected solution for individuals. However that is misleading, since, firstly, there are no defined patterns as fixed sequences of actions one can appropriate but patterns exist in an abstract way as effective niches in the encultured environment which are evolving constantly. Not every time one practice is following the other the same way to form the dedicated sequence, since in communities people act as self-regulated beings. Yet in large scale the patterned epistemic practices are repeatedly enacted in the practice community as probable sequences and the community members would rely on those patterns to achieve their goals. The community can benefit from patterns as solutions to the problems created by the community. The patterns are loosely connected across scales: any given pattern typically points to smaller scale patterns which can support it, and larger scale patterns in which it may participate (Alexander et al., 1977). Such pattern networks can allow designing support systems that facilitate patterned epistemic practices to be used systemically. For example, to support informal learning of individuals at workplaces, and to contribute to the organizational or community learning a self-organized feedback loop can be created using network of patterns (e.g. Ley et al., 2014, Tammets et al., 2014). Establishing the associations between discourse acts in Q\&A forums as patterned practices and seeing the interrelations between patterned practices as a network may be possible when addressing the discourse data using the social network analysis (SNA) approach modification (Kelsey, 2008) in which the discourse acts would be considered as nodes and the ties between discourse acts would be calculated from the frequency they are used in the system.

\subsection{Patterns of epistemic practices used by professionals}

Expertise and competence are developed through the participation in intensively functioning but unofficial communities of practice (Wenger, 1998). Q\&A forums of practitioners represent one form how such communities of practice can evolve and share expertise allowing individuals to learn informally. The 
research of professional expertise development in Q\&A forums usually focuses on the social network structures among different level experts and how they exchange expertise (Rissanen et al., 2010; Curran \& Abidi, 2006, Creswick \& Westbrook 2010, Rangachari, 2010). Few papers underline the importance of social processes with knowledge objects: content creation by users and expertise development of the participants (Santos et al., 2014; Schmidt \& Kunzmann, 2014; Cook \& Santos, 2014).

The papers referred to below frequently report of similar epistemic practices developed by different practice communities: sharing and developing experiences, implementing locally the new rules and localizing the practices, seeking and providing support and validating expertise. Different communities also reveal some particular cultural differences, how these common patterns of epistemic practices are manifested.

One the one hand, in the Healthcare sector, Anderson et al. (1987) reported that physicians' clinical patterns are influenced by a multitude of factors, one of the most important of which is their peers. The position of physicians in the consultation network significantly influenced their adoption and utilisation of new computer technology. Keating et al. (2007) found that physicians obtain information from colleagues with greater expertise and experience, as well as colleagues who were accessible based on location and schedule. Creswick \& Westbrook $(2007,2010)$ report clinical staff of tending to seek medication advice from members of their own profession, but some key individuals are used as sources of advice by all professional groups. Ankem (2003) reported of conferences being important for creating early awareness of innovative practices, while interaction with colleagues is the most important factor in stimulating use of an innovation among later adopters. Learning from other healthcare professionals experiences is common Coleman et al. (1957) wrote that a doctor will be influenced more by what his colleagues say and do in uncertain situations (e.g. when a drug is new), whenever and wherever they may occur, than in clear cut situations. Rangachari (2010) on the other hand showed that mainly explicit knowledge on general infection topics, rather than tacit knowledge on specific infection prevention practices was being exchanged among medical specialists.

Informal social discussions among healthcare specialists have been found to be important for routinizing new innovations. Lehoux et al. (2010) found that scientific arguments, clinical arguments and social arguments impact on social desirability and the routinization of new medical innovations. Social assumptions are developed by medical specialists who define social needs and preferences in ways they thought were appropriate given their patients' expectations. As medical specialists are members of society just like everyone else, their social assumptions may be the same as those of many other people (including patients). The socialisation of medical specialists reinforces the fairly unique position from which they determine which innovations are desirable or not.

On the other hand, the Construction sector is characterised by project work (often with cross-cultural group members), so that communication is an important issue within teams from the same company, but also as management of cooperation between different companies, levels of responsibility etc. (e.g. Baiden et al. 2006, Dainty et al. 2006). The work situations often include time pressure. Learning mainly takes place in the work process, and after the phase of initial vocational education it is mostly connected to the introduction of new products or processes. Different types of informal learning practices among professionals and in the professional networks were recently investigated by the Learning Layers project (Learning Layers D.1.1 \& D.1.2 see: http://learning-layers.eu/deliverables/). The empirical studies from the project revealed that requesting for help from other experts and actively searching people or documents to find a relevant source of knowledge are common to construction professionals' practices. We have observed how experienced craftsmen are sometimes reluctant to new knowledge, especially 
when it is brought to them by younger colleagues. As in other contexts, an important issue in construction communities is discovering experts who could be requested for help. Searching is often combined with collecting information, discussing experiences with other experts or distributing information to other experts in the network. Experts frequently validate their advice with experiences from different cases and often use metaphors to illustrate what they mean. Professionals need to adapt and communicate knowledge to the local context. For example: laws and regulations spread by the government are adapted into practical guidelines in some construction networks, which then are shared e.g. by newsletters. It is common to collect resources related to certain projects into a collection used for building site monitoring purposes. Some organisations join efforts to collect information of practices or problem issues (testing materials, creating schemas), and organize improving guidelines and regulations. In the Q\&A forum of Construction sector the most common problem types were found to be troubleshooting problems (Tammets et al., 2013).

Besides Healthcare and Construction sector we found research papers reporting of similar patterned epistemic practices among other communities of expertise. Vassilev et al. (2014) found that professionals in social networks are sharing knowledge and experiences in a personal community and accessing and mediating of resources. They highlighted that professionals' self-management support in social networks requires awareness of and ability to deal with network relationships: network navigation (identifying and connecting with relevant existing resources in a network), negotiation within networks (re-shaping relationships, roles, expectations, means of engagement and communication between network members), and collective efficacy (developing a shared perception and capacity to successfully perform behaviour through shared effort, beliefs, influence, perseverance, and objectives). These network mechanisms bring to the forefront the close interdependence between social and psychological processes and intertwine practical and moral dilemmas in identifying, offering, accepting, and rejecting support. Burchert and Schulte (2014) studied how apprentices in construction, rail traffic and insurance sector use open internet forums for apprentices. They found that students in such forums rarely discuss with regard to contents but rather use the platforms as place for organisational and social support, especially with reference to the final exams. The apprentices explained in interviews that they prefer to discuss professional issues with their local community of practice instead of strangers (in Germany, apprentices for two or three years parallely learn in schools and in VET companies).

Rissanen et al. $(2010,2014)$ reported from the studies in magician's forum that developing expertise requires not only systematic effort in learning from personal and collective experiences and improving various aspects of performance but requires efforts in tapping into cultural resources in the field, guidance from mentors, sharing professional know-how, helping to solve others' problems and brainstorming for new practices, networking, testing, transforming practice, improving performance, self-reflecting and analyzing it. They found in magician's professional network that the relations between professional expertise, advice-asking and reputation (nomination as a respected expert, nomination as a backward supporter) were attributed only to very central persons in network, whereas collaboration and informal interaction were more evenly distributed among different members of the professional community.

Rissanen et al. (2014) found professional magicians of not extensively talking and being secretive of their experiences (magic tricks) although this is the only way magician knowledge can be learned. The access to learning happened in asking questions and getting advice and was provided only to those who had established trustladen relations with experts. The expertise was tailored to fit the audience - main validation of experiences was feedback done in front of audience who validates the tricks (both ordinary audience and other magicians would provide validation to magic shows). It was common to develop own 
practices (new tricks), learning, appropriating, and modifying the practices of other fellows to develop novel implementations. Differently from healthcare and construction professionals, such uptake and modification behaviour was related with stealing and perceived as negative and unethical.

\subsection{Social recognition provision in professional forums}

In general formal recognition has often been used as a synonymous of authorized validation of knowledge, competences, practices or norms. The concept social recognition/validation (Heidegren, 2004) relates with peer-recognition or cultural recognition practices and is considered a basic medium for social integration. Social recognition is an accumulative validation process that creates social status to someone's identity (Heidegren, 2004). Through social recognition provision to the expertise and information that people share in the community, to the usefulness and reliability of their help-provision, and to their personal trustworthiness, professionals may gain perceived credibility in this community and be acknowledged as an authority, expert or trustee. The perceived credibility also relates with the resources these people create - the more reliable and useful is the information to the others, the more its author can be recognized. Jessen \& Jørgensen (2011) define 'perceived credibility' as the degree to which people believe that the information (and/or person) is perceived is credible when the following factors are combined: (1) Social Validation: a high number of people acknowledge a certain piece of information as trustworthy; (2) Personal profile: the members of the network provide an identity online (it can be edited in the same site or link to an existing profile, e.g. LinkedIn profile); (3) Authority \& trustee: a source of information is supported by an authority on the matter or trustees members. Construction forum analysis in the qualitative study of Tammets et al. (2013) indicated that person's credibility is highly evaluated by the help-seekers. Based on peer evaluations and status, users often turn to them or suggest asking from these users (e.g. I remember that user $X$ had similar experience).

In this paper we focus on social recognition as the socio-technically supported phenomenon in professional help-seeking forums. The main socio-technical functionalities used in forums for social recognition-provision are:

- endorsing people (e.g. LinkedIn.com);

- $\quad$ thanking people and seeing the number and list of thanks (ehitusfoorum.ee);

- (dis)liking content (in many forums);

- $\quad$ rating content with stars (in many forums);

- $\quad$ seeing person's status as karma and rating in the content and profile (habrahabr.ru), or seeing whether the user has been rated positively or negatively and why, for instance: fast and good answers; taught me as the amateur with the good advice; did a good job when building my house (ehitusfoorum.ee);

seeing the proportion overviews of person's activities with contents themes, topics and helpprovision (in many forums);

- badges given to persons based on forum practices (habrahabr.ru), or status or rank of the user such as construction veteran, construction pensioner, construction specialist (ehitusfoorum.ee);

- $\quad$ seeing the overview of persons' status in respect of the community (habrahabr.ru).

In our paper we explore the social recognition concept in association with an acknowledgement of the existence, validity, or legality of arguments provided in informal learning situations in Q\&A forums. Recognition as a validation may be: 
- the identification of something as having been previously seen, heard, known, etc. (validation with experiences) - relates with evidence-based learning, creating and maturing new practices;

- the acknowledgment of something as valid or as entitled to consideration (validation with rules, guidelines; validation with community practice, validation with the authority) - relates with credibility of information and people;

- the acknowledgment of achievement, service, merit, etc. (validation of other person's arguments, expertise, documents) - relates with social recognition provision, accumulated credibility, aggregated trustworthiness, social status and identity.

\section{Analysis of Q\&A professional discussion forums, a case study in Healthcare and Construction}

\subsection{Motivation}

The investigation of patterns of epistemic practices in professional Q\&A forums was motivated by several parallel research activities:

- Firstly, in Learning Layers project (http://learning-layers.eu) for developing tools to scale up workplace learning (Ley et al., 2014) there was a need to know what practices professionals in Healthcare and Construction sectors do naturally in informal learning settings of help seeking Q\&A forums. The results of this study can be used for informing the design process of tools for help seeking (Santos et al., 2014; Cook \& Santos, 2014) and maturing knowledge through localization practices (Mayer \& Schmidt, 2014; Schmidt \& Kunzmann, 2014).

- Secondly, our focus of looking the patterned epistemic practices using the network approach stemmed from the need to discover the interrelations between practices that could be used in designing the mutually interrelated informal learning tools. In the Learning Layers project a set of workplace learning tools to support capturing and organising knowledge in incidental learning, help seeking from the community, and maturing knowledge have been proposed (see Ley et al., 2014), that are integrated through the social semantic services which allow getting crowd knowledge based recommendations for discovering experts and learning content (Kowald et al., 2013). In such a socio-technical system different informal learning incidents (e.g. discovering some knowledge gaps at work, collecting useful information as part of work practice, requesting for help, providing help, socially recognizing credibility, summarizing and aggregating information, localizing and maturing rules and practices, sharing and uptaking practices) could be transferred from one pattern of epistemic practice to another in a synergetic way, promoting incidentally occurring practices of individuals to contribute to their personal learning and organizational or community learning.

- Finally, our attempt was to validate with the empirical data from the Q\&A forum the theoretical model of social recognition-provision that may be used as the basis for developing tools for workplace learning.

\subsection{Method}

\subsubsection{The dataset}

The domain focus of study in Learning Layers are the Healthcare (based in UK) and Construction (based in Germany). For this reason, the Q\&A forums analysed in this paper correspond to the same professional domain and countries. 


\section{The Healthcare forums in the UK}

Three forums from the Healthcare sector were analysed in the UK.

H1: The LinkedIn forum 'UK Nurse practitioners, Nurse prescribers, Practice Nurses network' (https://www.linkedin.com/groups/UK-Nurse-practitioners-Nurse-prescribers-4435682) is a discussion forum, but also its members use this space to share advertisements or 'hello' messages. Only those messages which contain questions, descriptions of problems, or petitions for further information have been selected. The total of threads selected are: 6 (with their corresponding answers). Only LinkedIn members can post. Range of dates: March 2013 - November 2014.

H2: 'MyHealthSkills' (https://www.myhealthskills.com/groups) is the forum for healthcare employees and aims 'to encourage likeminded people who are passionate about workforce transformation, learning and skills in the sector to network, to share best practice, expertise and resources.' This site includes 31 (open groups) / 66 (total of groups) and 35 closed groups (35/66). The following groups have been analysed due to their number of members, and because participants use the group to share questions: The open group 'dementia skills' have been selected because it is one of the open groups with higher number of participants (136 members). The open group 'Practice Education/mentoring' is the second group selected, with 46 members. The third group selected is 'Apprenticeships - Developing, implementing \& supporting apprenticeships' with 83 members. In general, participants use these groups mainly to share articles, news, blog posts, twitter posts etc. but in some occasions they share questions. A total of 4 threads were selected. It is not very common to share questions in this site. Posts from the years 2013 and 2014 were analysed.

H3: Practice nurses QA forum http://www.practicenursing.co.uk/forum/home.aspx is a forum intended for practicing healthcare professionals only, such as practicing nurses who work within GP Practices within Primary Care. There are 15410 members, from which 3500 have posted something. It contains 3 closed and 6 open forums and 3 forums in linked websites. We have analyzed publicly open forums "General discussion" and "New to practice nursing". The threads containing problems were selected, leaving aside social and advertisement threads. In total 4 long threads from time period April, 2010 - December, 2014 were analyzed, consisting of 208 posts, from which 49 were questions and 159 answers.

\section{The German Construction Forums}

Three forums were analysed:

C1: http://www.fachwerk.de/ is a forum specialised on renovating old houses which seems to have a wellworking community;

C2: http://www.bauexpertenforum.de/ is a forum for construction experts with (at the time of our analysis) 85.625 threads, 959.351 contributions and 26.924 registered users.

C3: http://www.haustechnikdialog.de/Forum/46/Haustechnikforum is a forum for craftsmen in house technique construction with 70.131 users, 2.127 .969 contributions and 1.892 .374 visits.

Threads on insulation (random sample) were chosen in order to get a first overview about the questions and response patterns in three different German forums on construction. Insulation is a very popular issue which concerns practitioners from different occupations (as well as house owners) and it allows different forms of reasoning (experiences with certain materials, physical laws, ethical considerations). In addition, threads were selected where the new German law on energy was discussed, where experts explicitly asked for other experts and where exchange of experiences was focused. All in all, 18 threads (six from each forum) with 243 contributions (questions and answers) from the years 2009-2015 were analysed. Most of the discussing persons were experts (professionals or experienced amateurs), but most questions were raised by novices who renovated a house in their leisure time. 


\subsubsection{Data analysis}

Three researchers analyzed the questions and answers in the professional forums. We used the qualitative content analysis method moving fom directed (for detecting the question types and the initial answer types) towards conventional analysis (for discovering the answer types) and for using data with networking approach the summative approach was taken on content analysis data (Hsieh \& Shannon, 2005).

For detecting the question types in the dataset we used the simplified question-type categories derived from Jonassen's (2000) typology of complex problems. Several of these question types have been reported in occurring in Q\&A forums of construction sector (Tammets et al., 2013). We simplified the question to four types based on forward- and backward reasoning (Sharma et al., 2012). In forward reasoning discourse acts in the thread take the form of the root of the search tree, rules that match to the root node's initial state are proposed, compared and validated until a configuration that matches the goal state is generated. The backward reasoning starts from the goal state, continues by generating the pool of possibly fitting rules that match with the root node, often new queries are required, and chaining backwards validates and eliminates the incorrect rules until start state is generated. Clinical reasoning has found to incorporate both forward- and backward reasoning (Stewart, 2004). The reasoning in construction sector Q\&A forums is reported of focusing extensively on troubleshooting (backward reasoning) problems (Tammets et al., 2013).

Question types:

- Rule-using (rule-based reasoning) - incorporate algorithmic and rule-using problems (e.g. How many $0,5 \mathrm{~kg}$ packages of glue I need for putting up 4 rolls of wall paper, each $10 \mathrm{~m}$ long? $0,5 \mathrm{~kg}$ pack lasts for 4-5 rolls; Where I can get the material with best price? Search online to find who sells it with best price!);

- Decision-making (forward reasoning) - incorporate decision-making-, dilemma problems (e.g. Should I use strategy $x$ or y to arrive this expected outcome? Using this strategy what the outcome(s) would be? Using strategies $x$ and $y$ would this satisfy expected outcomes?);

- Troubleshooting problems (backward reasoning) - incorporate troubleshooting- and diagnosissolution problems ( e.g. There is bad outcome (fault, disease), what causes it? Can this strategy/material/remedy be used for removing the fault/disease? Do these symptoms fit with this fault/disease?);

- Other type of questions.

For developing the classification of Answer types we followed the two step process. We started with the simplified discourse act categories used in scaffolding. During the analysis we discovered a number of more specific answer types that were considered as subcategories of providing information or validation. The researchers grounded the examples and formalized the names for those answer types (see Table 1). Thereby we expanded the answer categories to 31 categories. The full list of categories with examples is not provided due to limited space, but examples can be found from the results in section 4.

Table 1. Answer type categories in Q\&A forums

Initially used Answer Empirically found Answer types 


\begin{tabular}{|c|c|}
\hline types & \\
\hline $\begin{array}{l}\text { prompting for more } \\
\text { information or action }\end{array}$ & $\begin{array}{l}\text { prompting for more information, requesting for validation, requesting for } \\
\text { localization, heuristic questions }\end{array}$ \\
\hline $\begin{array}{l}\text { providing information } \\
\text { (without validation } \\
\text { elements) }\end{array}$ & $\begin{array}{l}\text { sharing opinion, sharing information, sharing experience, sharing new } \\
\text { practice, accumulating, summarizing info, reporting of uptaking the practice, } \\
\text { detecting the mistake or ambiguity, offering a troubleshooting plan, re- } \\
\text { defining the problem, correcting the mistake or ambiguity, recommending to } \\
\text { localize and implement, recommending to apply a rule, raising attention }\end{array}$ \\
\hline $\begin{array}{l}\text { providing validated } \\
\text { information }\end{array}$ & $\begin{array}{l}\text { validating other person, validating with guideline/rule/law, validating with } \\
\text { experience or expertise, validating with training info, validating with } \\
\text { community practice, validating with authority, validating as good practice }\end{array}$ \\
\hline other type of answer & other type of answer, joke, critique, feedback, de-escalation, no reply \\
\hline
\end{tabular}

From each forum questions $(\mathrm{Q})$ and answers (A) we created the dataset containing rows of sequential discourse act pairs (e-g. Q1-A1; A1-A2; A2-A3; Q2-A1 etc.) from all the threads in this forum. We also created summative data-sets for Healthcare $(\mathrm{H} 1, \mathrm{H} 2, \mathrm{H} 3)$ and Construction sector forums $(\mathrm{C} 1, \mathrm{C} 2, \mathrm{C} 3)$, and the Whole dataset (H1-H3, C1-C3). Next, we performed with these datasets the social newtork analysis (SNA), similar to the approach suggested by Kelsey (2008) using Gephi program (http://gephi.github.io/) to visualize the most frequent ties between $Q$ and $A$ as patterns of epistemic practices and to demonstrate what networks exits among these patterns of epistemic practices.

\section{Results: Patterned epistemic practices in professional Q\&A forums}

In the first phase of analysis we studied SNA figures (not presented in this paper) of patterns of epistemic practices in each forum and found that practices in some forums differed. Particularly we discovered that two Healthcare forums $(\mathrm{H} 1, \mathrm{H} 2)$ were rather used for sharing information, while in the $\mathrm{H} 3$ also the problem-solving practices appeared in the threads. The construction forums C1-3 were more alike. Our findings are supported by the assumption that patterns of epistemic practices are culture dependent (Alexander et al., 1977, Roepstorff, et al., 2010). Besides, patterns might offer solutions to common problems, and should re-occur in many contexts (Alexander et al., 1977).

Secondly, we explored separately Healthcare (H1-H3) and Construction (C1-C3) sector SNA figures of patterned epistemic practices (see Figures 2-3). We found that patterns of epistemic practices differed to some extent in Healthcare (they mostly discussed rule-usage problems, used validation with rules or authorities) and Construction sectors (they mostly discussed decision-making and troubleshooting problems, used validation with personal experience, and shared different figures, schemas). In the next subsections we present the summative pattern figures and observations discovered in Healthcare and Construction sectors.

\subsection{Patterns of epistemic practices in Healthcare forums}

Below the more frequent patterns (see figure 1) are presented with some examples of quotes extracted from the forums. Note that the practices in these patterns may not always appear in the same order, and some of them might be missing in different occasions. 


\section{- P1: Sharing information - validating other person's answer}

The pattern starts with sharing information. The answerer partially agrees/disagrees with answer or person. Validates previous answer as (in)correct/partially correct. Replaces with other answer or agrees with previous answer(or person), validates the answer by reformulating previous description.

Example:

- so Zostavax cannot be given to anyone who is on methotrexate or high dose ics? please give a simple answer my head hurts

- That's right NurseY! As a live vaccine best to discuss with Consultant for those pts on Methotrexate etc some will be happy for it to be given, others not. High-dose steroids - need to have 3-month gap between stopping steroids \& having any live vaccine - see NurseX's post on the "steroids \& shingles vaccine" thread

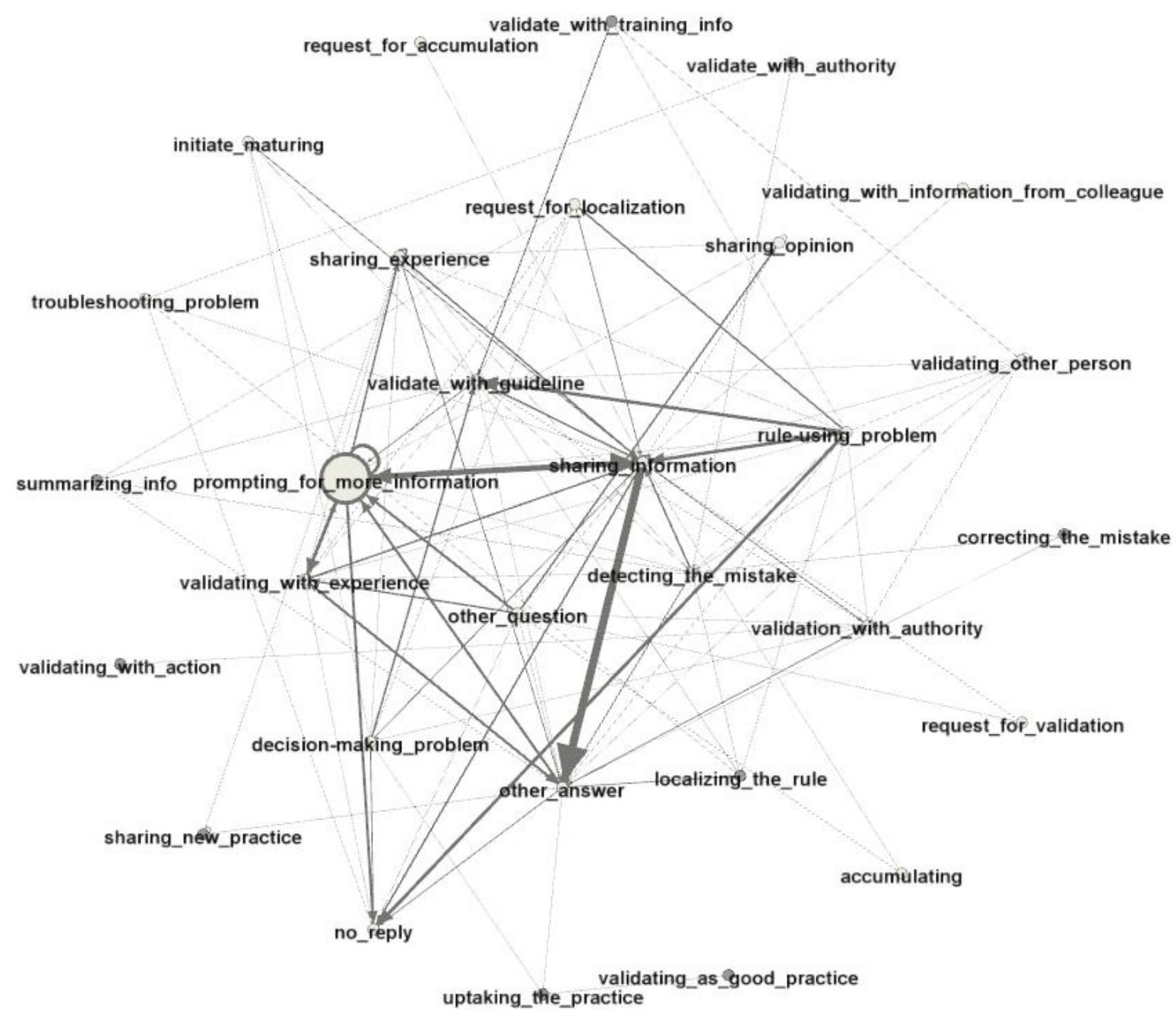

Figure 1. Patterns of epistemic practices in 3 British Healthcare forums (H1-H3)

- P2: Prompting for more info - sharing info - sharing experience - validating with experience - other answer - no reply 
Prompting for more information:

- $\quad$ Where in the UK....I am definitely interested, but I need to stay at my present job till next summer....so where are there openings. Are they permanent or temporary???

- Sharing info: give me a call xxx or drop me a email xxx to discuss further... we have shifts all over the UK; $x x x$ !

- Sharing experience: I am adult and children's trained nurse. What vacancies do you have? Validating with expertise:

- I am an HCA who will be a newly qualified assistant practitioner in December. I work in a gp surgery in $x x x$, bit would be interested in working in the newcastle area in the new year.

Other answer:

- Hiring Now : Oncology Nurses **Excellent Salaries on offer paid Tax Free*** Our Client a world class chain of Hospitals in xxx is hiring Western Educated and experienced Oncology Nurses, Best salary in the middle east plus excellent $T \& C$ on offer! Interested candidates please email CV to $x x x$

\section{- P3: Rule-using problem - validation with guideline/authorities or sharing information}

Rule-using problems:

- So we call all those who are 70 to 79 on 1 sept?

- Can you give Shingles with Pneumovax?

- Is it correct that HCA's can't give?

- What dose of steroid is "high dose" that would contraindicate live vaccines. trying to find info in green book and cant seem to see anything about what is deemed as high dose.

- Why cant HCA s give the shingles vaccine-assuming its because its a new vaccine. Is there a link $i$ can show to my gps- $i$ need some solid info if possible

Validate with the guideline:

- at least 6mth interval between live vaccines and chemo and would check with physician managaging care Link $x x x$

Validation with authority:

- NurseZ ....,I would contact specialist nursel doc/team for their oppinion as I have come across rheumatologists who very much have their own ideas and liaison re what they prefer with such patients and any shared protocols can be useful for all when dealing with further patients.

- Refer back to GP or manager. Don't stress. Divert calls and questions.

- I went to an immunisation update today and they said that is CAN be given with pneumovax and it is for those aged 70 or 79 on 1 st September. If they're not 70 until 2nd September they have to wait until next September. Also HCAs can't give it.

Sharing information:

- There is useful info on drug doses and immunosuppression in nathnac yellow book. Check it out NurseZ

- Nathnac yellow book box 3.4 corticosteroids and immunosuppression page 112 NurseZ. This useful table shows pred doses and equivalent doses of other steroids sandycat

- As promised link to $p d f x x x$

- We've had an email today....Clarification of eligibility for the Rotavirus vaccine Rotarix ${ }^{\circledR}$

- Have checked my e-mails at work today and I haven't got this latest e-mail. Has anyone else got it? 
$-\quad$ no

- $\quad$ had the same email too...

- $\quad$ we had email today saying not to give if born before 1 st of may

- P4: Detecting the mistake or ambiguity in rule - requesting for rule validation localizing/implementing the rule

Detecting the mistake/ambiguity in rule:

- $\quad$ LBC radio this morning interviewing a GP - missed the name. Told the listeners that Shingles vaccine available to all people over 70 from tomorrow. I texted and tweeted them to correct it but have not done so....

- we are getting people who have turned 70 since September who are booking in for shingles because the poster says 70 or 79 I have sent them away because they are not in the cohort but GP said today oh give them in they might have to wait until they are 79 now! did not seem interested in the PGD side of things.

- Getting a few patients who are now 80yrs but fall in the 1933/34 cohort I.e were 79yrs on sept 1st but have since turned 80yrs PGD/green book says eligible up to day before 80th birthday. Do we PSD these pts???

Request for rule validation:

- Just checking we still give to patients that have already had shingles. The spc doesn't state not to.

- Hi, does anyone know if there is any restriction on taking baby swimming after rotarix. Just querying cos know its shed in the stools?

Localizing/implementing the rule:

- Have had a patient on sulphasalazine who developed mild shingles after the vaccine. We checked before giving and sulsalazine not listed as a contraindication and I can't see getting shingles rash as a potential SE although obviously a live vaccine. Has anyone else come across this?? Patient is fine cos she knows we checked it all out before giving but I was wondering if I should Yellow Card it?

- the imm form site gives a range of dates of birth for eligable for the vaccine. we've decided to use this in our search criteria for patients

\section{- P5: Initiate maturing - sharing information - validate with guideline}

Someone has started the thread, and there may be several Q and A, then someone (an expert) sets this post as a sticky note and proposes to build all the Q and A around that. Adds relevant normatives to the sticky post. Example:

- Will make this thread a sticky before the multiple threads start. Can everyone try to confine their shingles questions to here in coming months. Following are the links to the gov.uk information, please add anything useful here.shingles programme

\section{- P6: Request for accumulating - summarizing info - accumulating}

Request for collective accumulation with rules how to do it is presented, summarizing data that were in earlier threads, summarizing info from past threads, creating a sticky post

- With your help I plan to list as many reference titles on Lads as I can. If you have any book recommendations then please reply to this post - I will add them to lads and then add them to the 
list here. Ideally include the ISBN in your post (this is a 9 or 13 digit number which is usually located near the bar code). If you see a book already listed here that you have read visit lads and add your star rating / review to help other members. I will start the list by going back through old posts for recommended books.

- I'm trying to recap all the information we have discussed over recent threads so they are easier to find, then will apply a sticky.

- $\quad$ P7: Sharing new practices - uptake of practice - validating as good practice

It is initiated without the request, usually appears in the end part of longer threads, where the norms have stabilized, may end with uptaking new suggested practice

- Even though the instructions say to aim the vaccine into the cheek, I've found that most babies take it better when it's given onto the tongue with the syringe resting on the bottom lip.I think it would be easier to give through a teat.

- $\quad$ i have found that giving it to mums to give without problem, i tuck a paper hanky under the chin and ask mum to point the syringe to the side of the mouth and syinge slowly. $i$ get on with the paper work while mum is doing this and so far have only heard chomp chomp chomp babies appear like it.

- Oh! I hadn't thought of that. I'll give it a go. Thank you...

- Good idea!

- Excellent idea..

- I've done this a few times recently - babes have taken it much better from mums than from me!

- yeah that's what I've been doing and seem to have worked very well..

- I tried that approach last year as it seemed quite reasonable and logical to me. I got such a frosty reception from one mother, and then a clueless response from another that I didn't bother anymore! Having said that, I even get some parents expecting me to undress the baby!

- I do it too, helps good

\subsection{Patterns of epistemic practices in Construction forums}

The Construction forums (C1-C3) show a number of patterns which do not (frequently) occur in Healthcare. The following figure 2 shows the accumulated patterns of epistemic practices.

Argumentation strategies that were found frequently in construction, but not as often in the Healthcare forums are:

- validation with chemical and physical laws, e.g. explanations of good and bad insulation constructions based on the dew point concept,

- critique on questions and answers (with direct de-escalation, agreement and jokes as counterpoints),

- re-definitions of the problem as considerations what is indeed necessary when a certain challenge is to be met;

- feedback as information what the questioner thinks about the answers and plans what he is going to do,

- troubleshooting plan as concrete suggestion how to proceed and basis for further discussion on concrete details, and

- heuristic questions as advices which were formulated indirectly (“have you already tried this...?”). 
Localization was picked up as recommendation to stuck to local experts, craftsmen, materials, literature etc. The most common epistemological patterns were the following (the questions and answers are written in German language, so that the examples were described instead of quoted directly):

- P8: Sharing information, prompting for more information and critique

This is the dominant pattern in the construction forums. This does not mean that the three forms always follow one after another: rather combinations of two are occurring. Interestingly, sharing information rather leads to prompting for more information than vice versa.

One example here is a thread where, as troubleshooting problem, BuilderX describes that he discovered mould in his insulation. He elaborates the concrete situation and the material he used. In reply to this, BuilderY prompts for some more information, he criticises BuilderX's construction work and shares information about an expert in the same forum whose contributions might be helpful. BuilderX answers to the question without reacting on the critique. In the following, BuilderY suggests a troubleshooting plan and criticises BuilderX again. The thread continues with critique on other constructions and with jokes about regional differences.

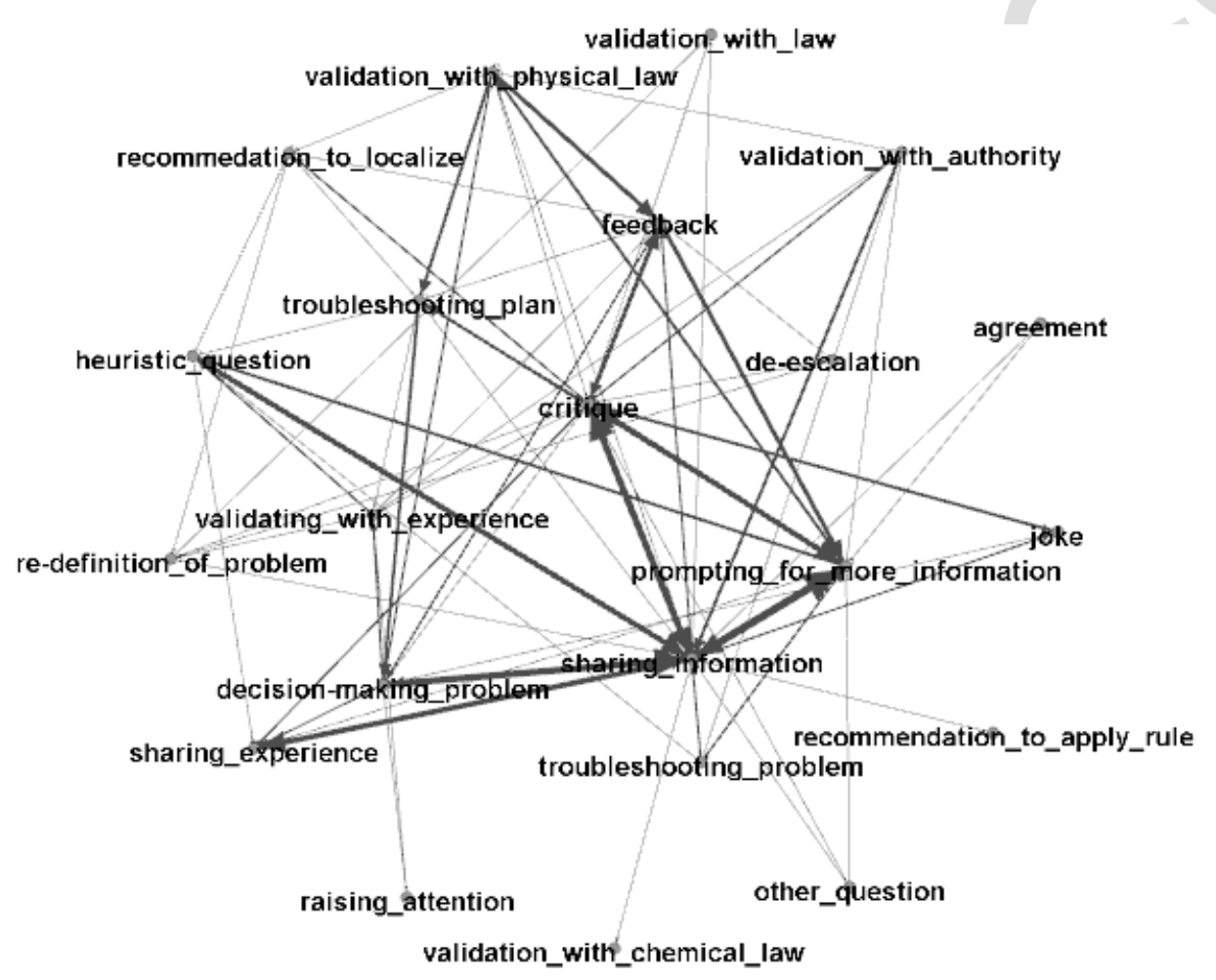

Figure 2. Patterns of epistemic practices in three German construction forums (C1-C3)

- P9: Validation with physical laws in the forums often leads to formulations of feedback or troubleshooting plans.

Example: BuilderZ asked how to make an insulation in an old house with certain specifications: he suggested to vary internal and external insulation. An architect replied by explaining and illustrating two relevant physical principles, the dew point and the thermal bridge, which make an alteration of internal and external insulation not effective and even problematic. Builder $Z$ then thanks and states that this answer was useful to him; he also sketches what he is going to do.

- P10: Decision making problems, sharing information and sharing experience. 
Usually only two of the three of these practices appear together. Interestingly, providing information and experience does not only resolve questions, but it leads to new decision making problems.

Example: BuilderG want to insulate the roof of his cellar. He describes the situation, which solution he excludes and what he plans. As an answer, BuilderR reports about experiences with his own cellar insulation, he gives some additional advice and explains his own construction perspectives. This leads to a new question from BuilderG, which is replied with more sharing of experience and information.

All in all, the discourse in the construction forum is characterized by attempts to clarify the problem by prompting for information, sharing information, critique, troubleshooting plans, heuristic questions, redefinition of the problem etc. The focus is on the problem, not on building up (positive or negative) relationships or stable communities; the development of communities is only a side-effect of problembased discussions. This underlines the findings of Burchert and Schulte (2014) that local communities of practice have a dominant role for learning of German craftsmen.

\subsection{Different problem-solving patterns across forums}

In the third step of analysis we observed the SNA figure (not presented) of patterned practices' from the whole dataset (C1-C3, H1-H3). Focusing on three general problem types we extracted from this figure the patterns of epistemic practices associated with rule-using, decision-making and troubleshooting type of problems (see figures 3-5). 


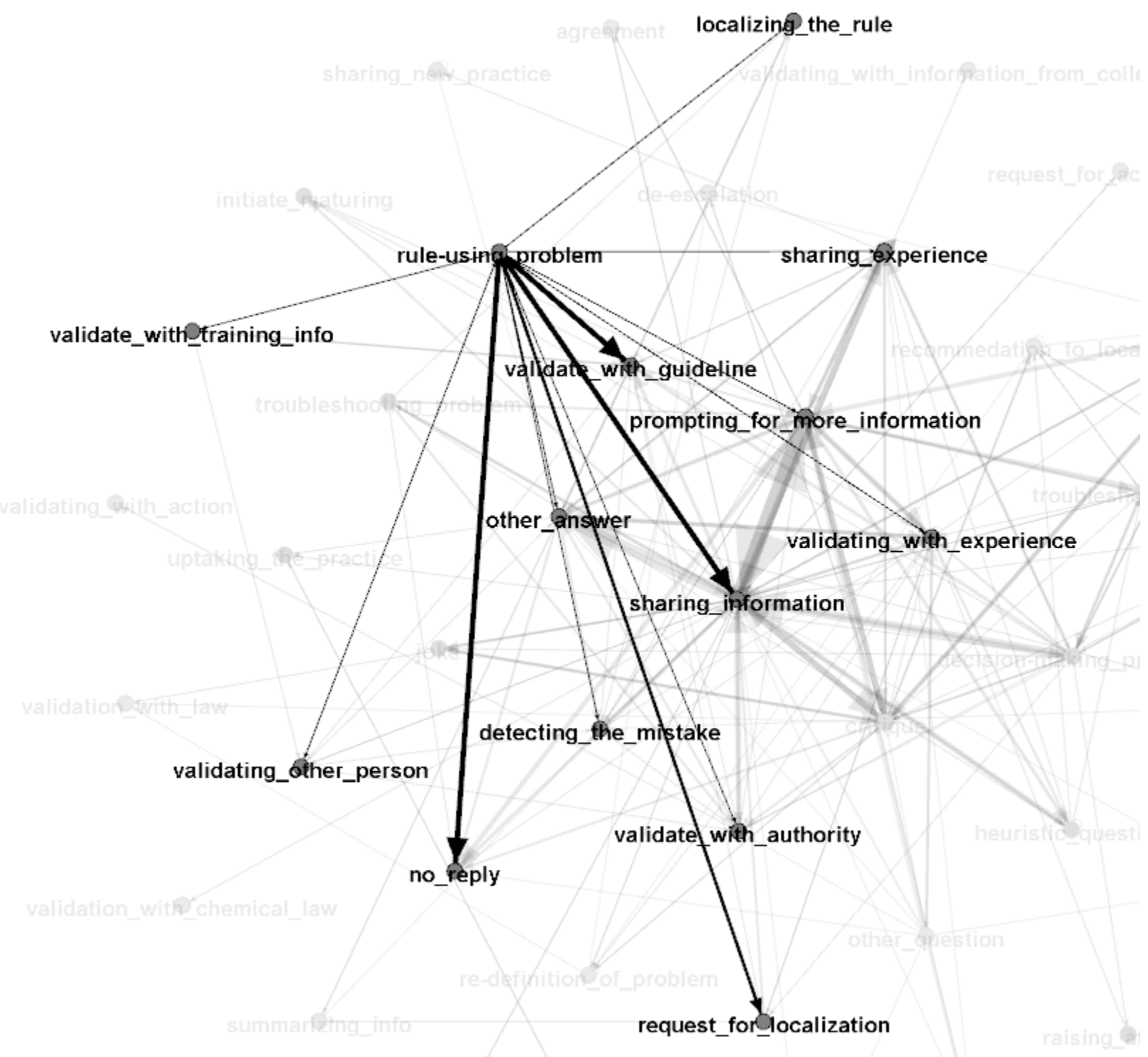

Figure 3. Rule using problem pattern based on 6 different forums data.

Rule-using problems, that can be solved by applying correct rules, were most common type of problems in Healthcare forums. In the longer discussion threads around new rules/guidelines we detected (see figure 3) the following network of patterns associated with rule-using problems (see also practice P1): the pattern of prompting for more information, sharing information and validating it with the rules and authorities (P2, P8), occurred after the guideline was launched, being accompanied by, the pattern of validating other persons answer (P1), the pattern of detecting mistakes/ ambiguities in the guideline and attempting localizing/implementing the rules (P4), and finally, usually in the second year, the pattern of sharing experiences and uptaking them appeared (P7). 


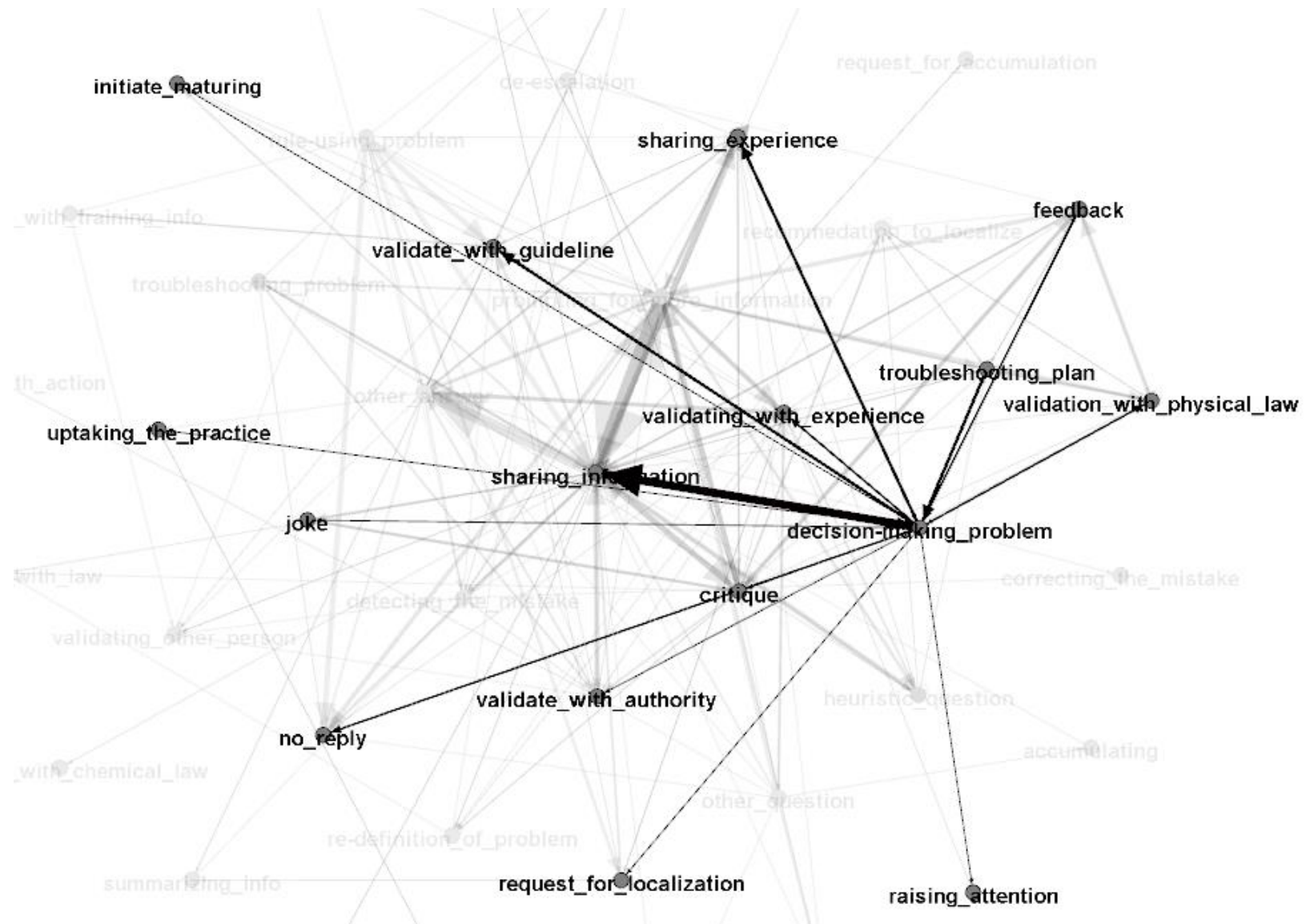

Figure 4. Decision-making problem pattern based on 6 different forums data

Decision-making problems that required comparing alternative solutions occurred mostly in the Construction forums. Several associated patterns of epistemic practices were found (see figure 4) forming the network of epistemic practices around decision-making problems (see also practice 10): sharing information, experiences and validating with experience, authority or physical laws pattern (P2, P8); has relations with the localization/locally implementing request pattern $(\mathrm{P} 4)$, maturing pattern (P5), and sharing and uptaking the new practice pattern (P7). 


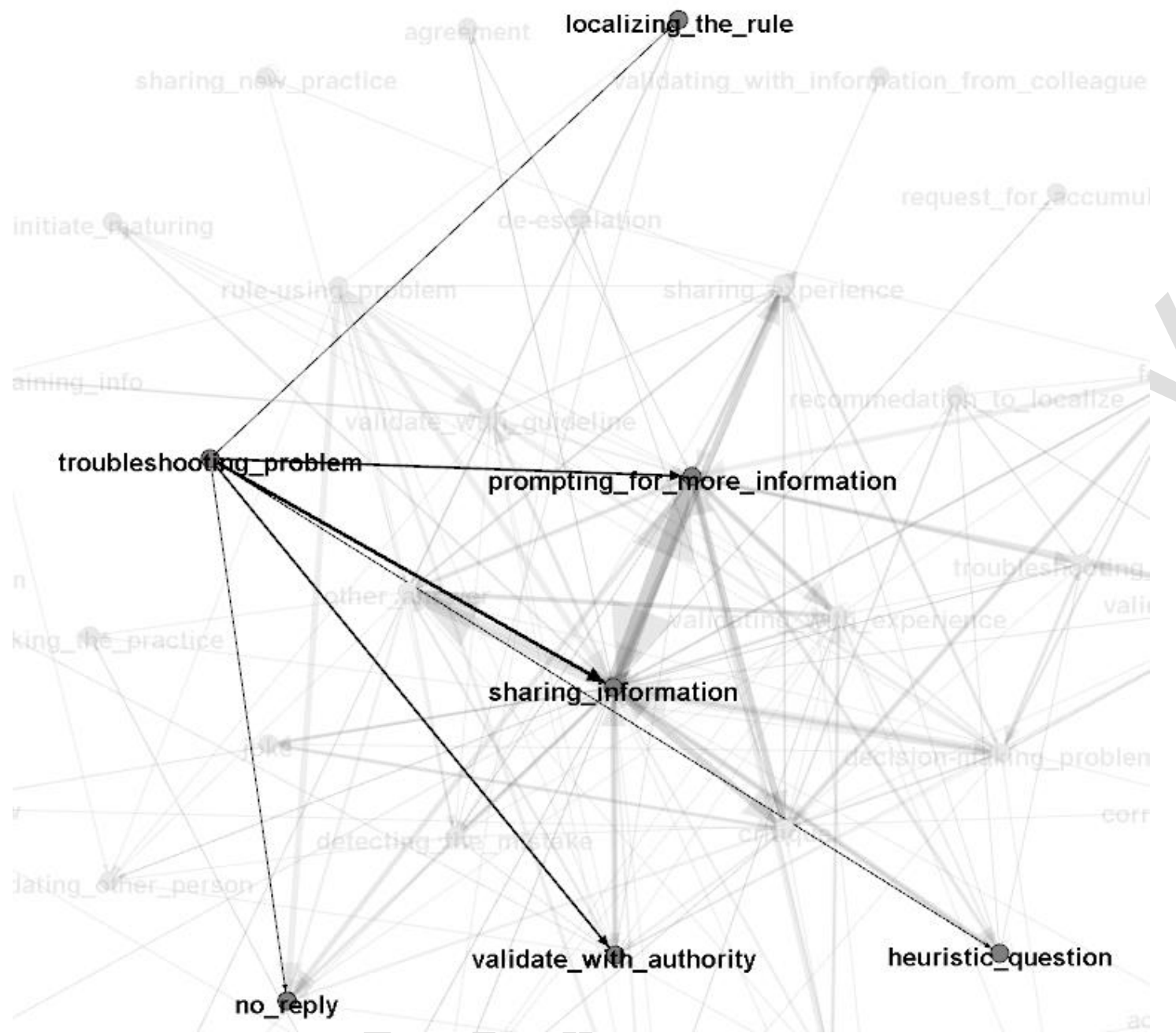

Figure 5. Troubleshooting pattern based on 6 different forums data

Troubleshooting problems require testing out different options to rule out what the problem is caused by. The troubleshooting discussions are often intense and involve sharing information of the problem state as well as prompting for more information, suggesting approaches how to proceed and validating these suggestions with rules or authorities. The troubleshooting pattern networks (see figure 5) contain patterns of prompting for more information (P2), and localizing/implementing the rules (P4).

\section{Discussion: Enhancing the quality of informal learning in professional help-seeking communities}

We can summarize the main findings from the SNA analysis from epistemic practices in professional Q\&A forums as follows:

- Informal Q\&A forums of professionals may have specific discussion cultures, some forums do not discuss problems but rather use forum for sharing information and socializing.

- Patterns of epistemic practices in Q\&A forums of Healthcare and Construction are different in respect of which problem types are prevailing in the domains - rule-using problems prevail in Healthcare, decision-making and troubleshooting problems in Construction forums. 
- Different practice communities use to some extent common patterns of epistemic practices:

$\circ$ problem-solving using social recognition practices (see figures 3-5 for networked practices in problem-solving)

$0 \quad$ sharing new practices and experiences and uptaking practice $(\mathrm{P} 7)$

- localizing and maturing rules/guidelines (P4)

- Problem-solving patterns incorporate also other patterns of epistemic practices related with prompting for and sharing experiences $(\mathrm{P} 2, \mathrm{P} 8)$, uptaking practice $(\mathrm{P} 7)$, localizing $(\mathrm{P} 4)$ and maturing rules and guidelines (P5).

- The pattern of validating other person's answers (P1) did not come out as an important element in the decision-making and troubleshooting types of problems.

- The informal Q\&A forums contain some examples of learning practices:

- learning from others by being collectively helped to solve problems;

- learning by observing how a problem was solved collectively (or by reading which possible solutions were suggested);

$\circ$ discovering new practices at work and sharing those with others (especially in Construction also by using negative examples);

○ uptaking new practices shared in the forum;

- learning how to define a problem properly;

- learning how to raise the right questions.

Based on the theory presented in section 2.3 we have constructed the model of social recognition provision (see figure 6). This model uses the social recognition mechanisms in the Q\&A forums as central and relates social recognition also with the external patterns of epistemic practices of: (1) practicing and learning at work, and (2) formalizing practices into guidelines, rules and norms in authorized bodies. The social recognition process is used for creating credibility for persons (status, expertise) and content (practice examples, help comments) produced by these persons. The practice examples brought to the forum for social recognition are often validated using the rules, norms and guidelines, the authorities or the personal/community practice to support the arguments. Part of the social recognition processes is an interrelated mechanism of validating the user/content that usually works as a socio-technically supported mechanism using technical functionalities that relate content status with user status. The practice examples are also brought to social recognition for validating the mistakes in the norms and guidelines or describing the localization of guidelines. This allows norms, rules and guidelines to be collectively matured that may be used for updating the rules based on practice and enables responsiveness or organizations and professional communities to internal and external changes. The Q\&A forums also serve as the arena for reporting of new practices and uptaking the socially recognized practices and using them at workplace.

Our empirical findings support this social recognition provision. Particularly we found several types of validation practices in $\mathrm{Q} \& \mathrm{~A}$ forums being important components in accumulating socially the credibility of supportive answers to different types of problems. Validation practices are an important part of social recognition provision and also could increase the credibility of professionals as experts in the informal professional communities that we did not explore in this study. The validation of other person's answers, that according to the social recognition model increases persons' status was not frequently observed at Construction forums, but appeared in Healthcare forums. We found that solving rule-using and decisionmaking types of problems may trigger patterns of localization and maturing, and sharing and uptaking 
practices - both relate with noticing and bringing personal workplace learning experiences to be approved through the social recognition mechanisms. In the Construction forums, questions of problem definition and critique were important to clarify to which problem or decision exactly the solution should fit.

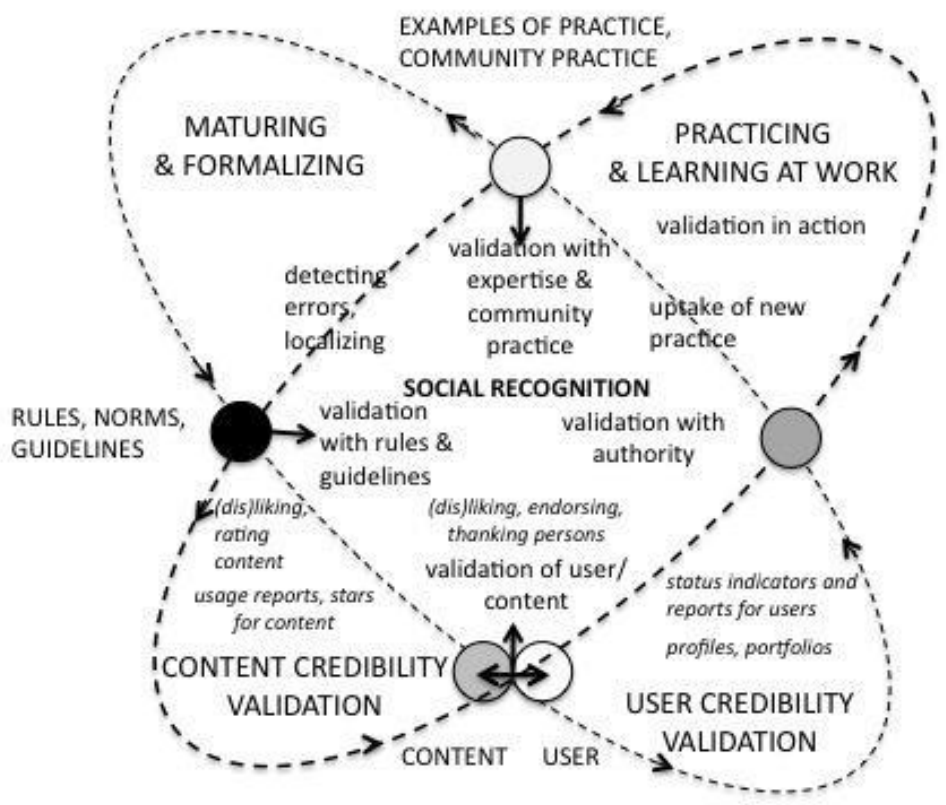

Figure 6. A model of social recognition provision in professional Q\&A forums

Based on our findings, validated in the light of the social recognition provision model we suggest that in order to enhance informal learning and maturing organizational knowledge in Q\&A forums, the following aspects might be facilitated with socio-technical functionalities:

- the information validation practices:

- validating with rules

$\circ$ validating with authority

$\circ$ validating with personal practice experiences/ community of practice

○ validating with taking (collective) action

- the validation of other person's answers (accumulating expertise to persons).

Organizational learning (maturing of rules/practices) could be improved by triggering the following practices in Q\&A forums and relating these with previously mentioned validation elements:

$\bigcirc \quad$ learning how to define different types of problems;

- accumulating (giving rules how to do it collectively);

○ summarizing;

- reporting of new practices;

- requesting-proposing localization/implementation of rules;

$\circ$ reporting of mistakes/ambiguities in applying the rules/practices.

Some limitations of our empirical study are that we did not clarify what the meaning of discovered patterns of epistemic practices is for the practice communities - we cannot state clearly which problems 
these practices solve for the community. Secondly, we limit our model application remarking that not all the described social recognition elements might be useful and approved in every kind of communities. For example, small closed work communities might be reluctant to revealing person's status as well as getting negative ratings to the content they create, but these socio-technical functionalities for social recognition may be well working in large informal practice communities.

\section{Conclusion and Future Work}

This paper studied the epistemic practices of two professional domains (Healthcare and Construction) by analysing a collection of public Q\&A forums of practitioners that are used for help-seeking and informal learning. We used the SNA visualizations created from the sequences of practice patterns and the examples from the discourse to illustrate the patterns of epistemic practices which incorporate social recognition. Another contribution from this paper is a model of social recognition, which captures the different steps of an accumulative validation process in informal practice communities. Our future work is directed towards using the resulted patterns of epistemic practices and the social recognition model to design and validate with workplace users the scaffolds for informal learning discussion and collaboration in a professional networking environment. For example, we are exploring how to support bringing the issues arising in Q\&A forums to be entering to the structurally supported maturing process.

\section{References}

Alexander, C., Ishikawa, S., Silverstein, M., Jacobson, M., Friksdahl-King, I., Angel. S. (1977). A Pattern Language: Towns, Buildings, Construction (Center for Environmental Structure Series). Oxford University Press.

Alexander, J. C. (1988). Action and its environments. Toward a new synthesis. New York Columbia University Press.

Anderson, J.G., Jay, S.J., Schweer, H., Anderson, M., Kassing, D. (1987). Physician communication networks and the adoption and utilization of computer applications in medicine. In: Anderson JG, Jay SJ, editors. Use and impact of computers in clinical medicine. New York: Springer. 185-199.

Ankem, K. (2003). Influence of information sources on the adoption of uterine fibroid embolization by interventional radiologists. Journal of the Medical Library Association 91(4): 450-459.

Baiden, B.K., Price, A.D.F. \& Dainty, A.R.J. (2006).The extent of team integration within construction projects. International Journal of Project Management, 24(1), 13-23.

Bray, D.A., Konsynski, B., Thomas, D. (2007). IS-Driven Organisational Responsiveness. Emory University. Available at: http://papers.ssrn.com/sol3/papers.cfm?abstract_id=984598

Brusoni, S., Prencipe, A., Pavitt, K. (2001). Knowledge Specialization, Organisational Coupling, and the Boundaries of the Firm: Why Do Firms Know More Than They Make? Administrative Science Quarterly, 46, 4, 597-621. Johnson Graduate School of Management, Cornell University.

Burchert, J. \& Schulte, S. (2014): Die Nutzung des Internets in der dualen Ausbildung. Eine berufspädagogische Betrachtung auf Basis empirischer Forschungsergebnisse. Peter Lang, Frankfurt a.M.

Coleman, J., Katz, E., Menzel, H. (1957). The diffusion of an innovation among physicians. Sociometry 20(4): 253-270.

Cook, J. and Santos, P. (2014). Social Network Innovation in the Internet's Global Coffeehouses: Designing a Mobile Help Seeking Tool in Learning Layers. in Educational Media International. 
Creswick, N., Westbrook, J.I. (2007). The medication advice-seeking network of staff in an Australian hospital renal ward. Studies in Health Technology and Informatics 130: 217-231.

Creswick, N., Westbrook, J.I. (2010). Social network analysis of medication adviceseeking interactions among staff in an Australian hospital. International Journal of Medical Informatics 79(6): e116125.

Curran, J., Abidi, S.S.R. ( 2006). Evaluation of a discussion forum for knowledge sharing among emergency practitioners: A social network approach. Source of the Document Studies in Health Technology and Informatics 124, 941-946

Daft, R.L, \& Weick, K.E. (1984). Towards a model of organisations as interpretation systems. Academy of Management Review, 9, 284-295.

Dainty, A., Moore, D. \& Murray, M. (2006). Communication in construction: theory and practice. London: Taylor \& Francis.

Goodstein, J. (1995). Employer involvement in eldercare: An organisational adaptation perspective. The Academy of Management Journal, 38(6), 1657-1671.

Heidegren, C.-G. (2004). Review essay. Recognition and social theory. Acta Sociologica, 47(4), 365-373.

Hutchins, E. (2010). Cognitive ecology. Topics in Cognitive Science, 2, 705-715.

Hsieh, H.-F., Shannon, S.H. (2005). Three approaches to qualitative content analysis. Qualitative Health Research, 15(9), 1277-1288.

Jacobs, C. 2003. Managing Organisational Responsiveness - Toward a Theory of Responsive Practice. Wiesbaden: DUV.

Jessen, J., \& Jørgensen, A. H. (2011). Aggregated trustworthiness: Redefining online credibility through social validation. First Monday, 17(1).

Keating, N.L., Ayanian, J.Z., Cleary, P.D., Marsden, P.V. (2007). Factors affecting influential discussions among physicians: a social network analysis of a primary care practice. Journal of General Internal Medicine 22(6), 794-798.

Kelsey, S. (2008). Handbook of Research on Computer Mediated Communication. IGI Global.

Kowald, D., Dennerlein, S., Dieter, T., Walk, S. and Trattner, C. (2013). The Social Semantic Server - A Framework to Provide Services. In Social Semantic Network Data, I-Semantics, Graz, Austria.

Learning Layers D.1.1 \& D 1.2. Available at: http://learning-layers.eu/deliverables/

Lehoux, P., Denis,J-L., Rock, M., Hivon, M. \& Tailliez, S. ( 2010). How medical specialists appraise three controversial health innovations: scientific, clinical and social arguments. Sociology of Health \& Illness 32 (1) 2010, 123-139

Ley, T., Cook, J., Dennerlein, S., Kravcik, M., Kunzmann, C., Pata, K., Purma, J., Sandars, J., Santos, P., Schmidt, A., Al-Smadi, M., Trattner, C.(2014). Scaling informal learning at the workplace: A model and four designs from a large-scale design- based research report. British Journal of Educational Technology, 45(6), 1036 - 1048.

Maier, R., Schmidt, A. (2014). Explaining organizational knowledge creation with a knowledge maturing model. Knowledge Management Research \& Practice, vol. 2014, 1, pp. 1-20

Nonaka, I. \& Takeuchi, H. (1995). The knowledge-creating company. New York: Oxford University Press.

Parsons, T. (1937). The structure of social action. New York: Free Press.

Pata, K. \& Bardone, E. (2014). Promoting distributed cognition at MOOC ecosystems. In: Lecture Notes in Computer Science (LNCS). Learning and Collaboration Technologies. Technology-Rich 
Environments for Learning and Collaboration. Part I.: HCII International, LCT 2014, Crete 2227.06.2014. (Eds.) Panayiotis Zaphiris, Andri Ioannou. Springer, 204 - 215.

Rangachari, P. (2008) Knowledge sharing networks related to hospital quality measurement and reporting. Health Care Management Review 33(3): 253-263.

Rissanen, O., Palonen, T., Hakkarainen, K. (2010). Magical expertise: An analysis of Finland's national magician network. In Dirckinck-Holmfeld L, Hodgson V, Jones C, de Laat M, McConnell D \& Ryberg T. (Eds.) Proceedings of the 7th International Conference on Networked Learning 2010.

Rissanen, O., Pitkänen., P., Juvonen, A., Kuhn, G., Hakkarainen, K. (2014). Expertise among professional magicians: An Interview study. Frontiers in psychology, 5, 1484, 1-13.

Roepstorff, A., Niewöhnerc, J. \& Beck, S. (2010). Enculturing brains through patterned practices. Neural Networks 23 (8-9), 1051-1059.

Santos, P., Cook, J. Treasure-Jones, T., Kerr, M. \& Colley, J. (2014). Networked Scaffolding: Seeking Support in workplace learning contexts. Proceedings of the 9th International Conference on Networked Learning 2014 (Edinburgh, UK).

Schmidt, A., Kunzmann, C. (2014). Designing for knowledge maturing: from knowledge-driven software to supporting the facilitation of knowledge development. In: International Conference on Knowledge Management (I-KNOW 2014), ACM.

Sharma,T., Tiwari, N., Kelkar, D. (2012). Study of difference between forward and backward reasoning. International Journal of Emerging Technology and Advanced Engineering Website: www.ijetae.com, 2, (10), October 2012.

Stewart, T. M. (2004). Teaching the art and science of plant disease diagnosis: Training students with DIAGNOSIS for Crop Problems. The Plant Health Instructor. Available at: DOI:10.1094/PHI-T2004-0426-01

Tammets, K., Pata, K, Laanpere, M. (2013). Promoting Teachers' Learning and Knowledge-building in the Socio-technical System. The International Review of Research in Open and Distance Learning, 14(3), 251 - 272.

Tammets, K., Laanpere, M., Ley, T., Pata, K. (2013). Identifying problem-based scaffolding patterns in an online forum for construction professionals. In: Proceedings of the 8th European Conference on Technology-enhanced Learning: European Conference on Technology-Enhanced Learning. (Eds.) Hernández-Leo, D., Ley, T., Klamma, R., Harrer, A. Heidelberg: Springer, (Lecture Notes in Computer Science), 526 - 531.

Tynjala, P. (2008). Perspectives into learning at the workplace. Educational Research Review, 3(2), 130154.

Vassilev, I., Rogers, A., Kennedy, A. and Koetsenruijter, J. (2014). The influence of social networks on self-management support: a metasynthesis BMC Public Health, 2014, 14:719.

Wenger, W. (1998). Communities of practice: Learning, meaning, and identity. Cambridge: Cambridge University Press.

Werquin, P. (2010). Recognizing non-formal and informal learning. Outcomes, policies and practices. OECD 2010. Available at: http://www.eucen.eu/sites/default/files/OECD_RNFIFL2010_Werquin.pdf 